\title{
A Practical Solution for Location Estimation in Manually Deployed Wireless Sensor Networks
}

\author{
Om Prakash Sahu', Tarun Dubey ${ }^{2}$ \\ ${ }^{1}$ Department of Electronics and Communication Engineering, National Institute of Technology, Kurukshetra, India \\ ${ }^{2}$ Department of Electronics and Communication Engineering, Invertis University, Bareilly, India \\ E-mail:ops_nitk@yahoo.co.in, tarundubey79@yahoo.co.in \\ Received February 15, 2011; revised March 9, 2011; accepted March 15, 2011
}

\begin{abstract}
This paper addresses the existing research and adds another aspect of functionality by incorporating pertinent sensor nodes to provide a dynamic location discovery and estimation. The software used provides an easy graphical user interface to visualize a particular location in accordance with geographical latitude and longitude. A simple real time location estimation technique is worked out for wireless sensor networks based on manual deployment of sensors. The proposed scheme finds more efficient solutions with less quantity of sensors as compared to existing deployment schemes. The set up is evaluated exclusively in real environments using IRIS sensor nodes supported by a global positioning system module to provide visualization of an outdoor location. The results are offered by Google Earth application.
\end{abstract}

Keywords: Wireless Sensor Network (WSN), Global Positioning System (GPS), Localization, Sensor Node, IRIS Sensor

\section{Introduction}

Wireless sensor networks have been identified as one of the most prominent technologies for the 21st century [1]. With upcoming advancements, sensor networks find more prosperous grounds to spread in areas where traditional networks fail or are inadequate. They find applications in a variety of areas such as climate monitoring, military use, industry and sensing information from inhospitable locations. Sensor networks are also being used for conditional based maintenance, surveillance, computer augmentation and inventory tracking. Many other applications are yet to appear when the technology will become more widely available. Unlike other networks, sensor networks depend on deployment of sensors over a physical location to fulfill a desired task. Dense deployments imply the use of hundreds or thousands of sensor nodes in small areas with tight coupling within their environments to ensure effective coverage range in a geographical field. Sensor networks also have various computational constraints like range management, topology selection, power consumption and node localization. Although a lot of research is being conducted, there are several open issues for sensor networks such as signal processing [2], deployment [3], operating cost, localization and location es- timation, since the potential for sensor networks in diverse fields has been realized, various software frameworks are being developed to make deployments relatively easy in terms of configuration, maintenance and usability.

In this paper we present a solution for estimating the location of sensor nodes in a manually framed WSN by using Mote View 2.0.1F software. The software has a complete computational stack and provides a reliable IRIS sensor platform for monitoring the location of the manually placed nodes in terms of latitude and longitude values. The software architecture provides a customized application with minimum errors. The nodes are executed on a computer through a gateway connected to it. The system is also equipped with Google Earth application for enabling visualization.

The IRIS sensor nodes have open access to the outdoor environment as they are placed manually on a roof top of a building; the exact location of the building or site is obtained in terms of relative latitude and longitude. Indoor applications are not successful as the system requires massive optimization to minimize performance degradation due to walls and other objects found in indoor environments because of the use of a GPS device. This paper strongly focuses on localization and suggests a simple solution to localize sensors in a manually deployed 
WSN.

\section{Related Work and Background}

Recent research in WSNs strongly focuses on sensing applications, unlike the applications that were proposed in the previous years. The current perspective to deploy sensors manually is used in several projects for real system deployments [4]. Our proposed solution has minimum overhead and it is cost effective interfacing between different networks is gaining importance as research on WSNs related software environments is being pursued during the last few years [5]. There are sources providing references to a number of related solutions, some of these approaches are quite similar to small sensor networks with large systems. However, most of the existing softwares deal with respective application scenarios and target domains. They choose a different architecture for solving similar problems like fault detection and configuration of the network in real time [6]. Some configurations follow an N-tier architecture $[7,8]$ while others extend this concept using a peer-to-peer logic [9] recently, BSPA was presented, in which some nodes in the network are first selected and then formed into a backbone network [10]. Methods to adjust the directions overlap with the local coordinate systems and an approach to merge them has also been proposed to resolve the ambiguities. A graphical virtual instrumentation development platform to check data flow and interface for real time data processing has also been proposed [11].

For industrial and academic research, the inherent interaction between sensor networks and the physical world makes location awareness as one of the essential services for emerging applications such as location based directory service [12] and entity tracking [13]. In addition to the above work, a solution of walking GPS [14] is suggested for use in real manual deployments of sensors to evaluate the localization performance but projects some errors within the range of few meters. In view of the above cited limitations that are exhibited by current localization techniques, we have proposed a practical solution to provide better accuracy in a running network with less complexity, minimum sensors and meek cost.

\section{System Description and Overview}

The architecture of WSN, as shown in Figure 1, consists of spatially distributed sensor nodes, which form a multihop network to measure the parameters of interest within the application domain. They communicate it to a sink node or base station for access by the end user. Software framework developed, makes deployments relatively easy to configure and maintain. Mote View 2.0.1F addresses this issue of location estimation in a fine grained fashion, having a set of tools to control the network and provide data related to latitude and longitude. As described earlier, a network of self powered IRIS sensor nodes is deployed in a three-dimensional space. A monitoring or a sink node is responsible for collection of data reports form individual sensor nodes is directly connected with a personal computer. We do not assume any specific routing or MAC protocol [15] for this network, hence each node along with the sink, which has a unique ID, and knows its location within the network. The nodes configured in the network allow data transmission in hops from one node to the other within the network and finally to the base station or sink connected with the computer. The transmission from nodes to the sink can take any possible path, because if any node fails or disables, the data flow is not interrupted. Mote View 2.0.1F developed by Crossbow [16] is used to program the nodes for displaying the real time data to estimate the location using Google Earth application, an online available software tool.

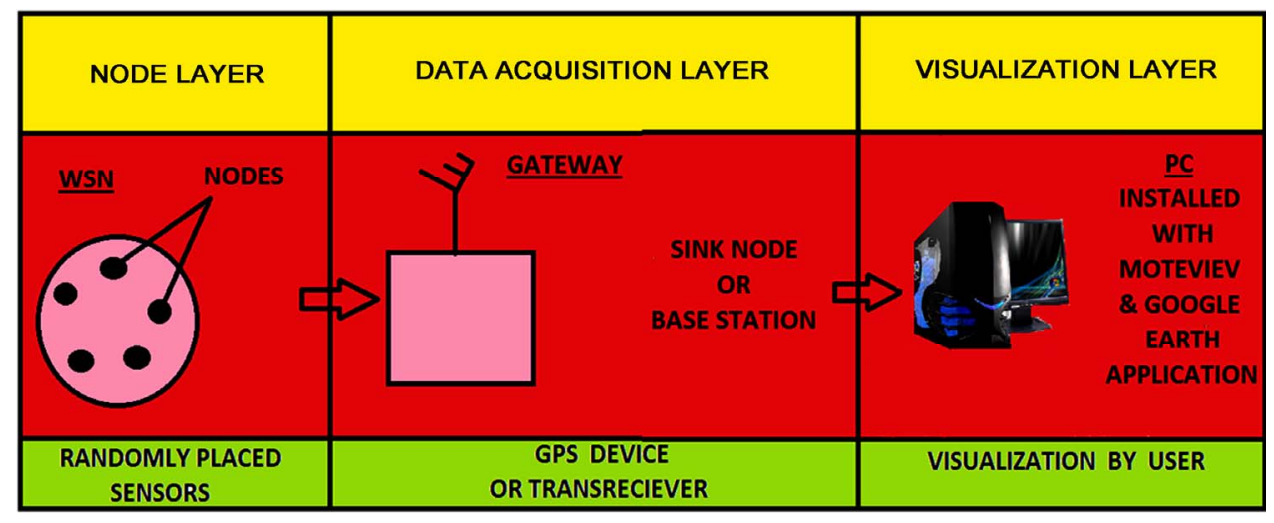

Figure 1. System description of a manually deployed wireless sensor network. 


\section{Performance Evaluation and Deployment}

A self-powered IRIS sensor node consists of a mote (processor and radio) and a sensor board, programmed with a unique node ID, is enclosed in casings and physically placed at different locations. A laptop or a personal computer interfaced to gateway (MIB520) provides data connectivity to the server through a port. The IRIS sensor platform provides a wider coverage area, low power consumption and better optimization for several other applications and is based on IEEE 802.15.4 compliant radios.

With an internet connection available, the user can control the system remotely from any location as the nodes are programmed and capable to transmit message packets with a header. The program running on the server collects and displays information of interest to the user through a wireless link. In order to achieve real time live data from sensor nodes, the base station has an inbuilt GPS antenna to transmit the real time information of latitude and longitude points of the nodes placed on the rooftop of the building. The gateway also allows bidirectional communication to organize the current information of a specific geographic location (longitude and latitude). To visualize the location in a 3D space, altitude can also be incorporated as a third parameter but is not automatically generated by the software under use, since most environments use 2D representations, due to the advantage of simultaneous display and network spanning across individual buildings.

\section{Experimental Results}

The Mote View platform [17] enabled to administer the nodes in terms of specific values for latitude and longitude. The deployed IRIS sensor motes with sensing modalities monitored the exact geographical location of the individual building in terms of relative positions for latitude as $28^{\circ} 29^{\prime} 2217^{\prime \prime} \mathrm{N}$ and longitude as $79^{\circ} 49^{\prime} 4267^{\prime \prime}$ E. The specific location of the site in instantaneous time span and spatial form was correctly visualized with Google Earth application through the site's satellite image with live data. The satellite image of the site where the wireless sensor network was tested can be seen in Figure 2. A perfect match between the deployment location and relative geographical position was observed. However localization errors are not provided due to irregular deployments and device limitations.

\section{Conclusions and Future Scope of Work}

The technique to deploy sensor nodes manually is currently used in several projects, and there are scenarios of real system deployments, where manual deployment is the only solution. Results show that the deployed nodes estimate their relative latitude and longitude positions for a reference point. The average localization error is mainly due to the limitations of the devices used, because for location information, the nodes completely rely on the global positioning system and localizing themselves in the middle of their proximate placements or reference points.

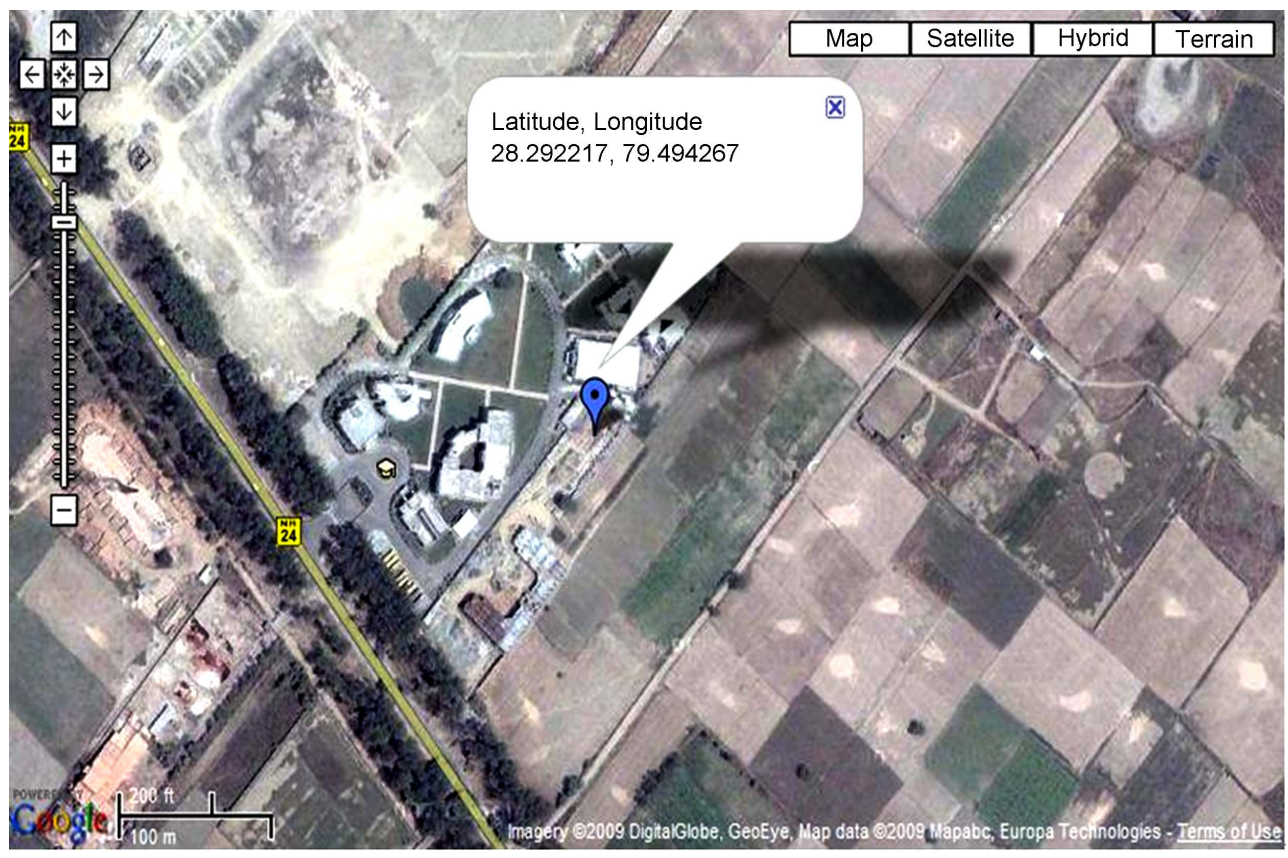

Figure 2. Screen shot image of the site obtained through Google Earth application. 
In future, performance evaluation and scalability will be done through simulation. The experience from the current deployment of the sensors can be used further to address the aerial deployment. Considering the rate, altitude, and trajectory of sensor nodes the actual location information at the time of initial deployment, can also be obtained using our solution, giving a starting point towards a better and precise localization scheme. The authors are working towards making it more common and adaptable for other user communities also.

\section{Acknowledgements}

The authors would like to acknowledge the support and assistance of Electronics and Communication Engineering Departments at National Institute of Technology, Kurukshetra and Invertis University, Bareilly.

\section{References}

[1] D. Culler, D. Estrin, and M. Srivastava, "Overview of Sensor Networks,” IEEE Computer, Vol. 37, No. 8, 2004, pp. 41-49.

[2] G. J. Pottie and W. J. Kaiser, "Embedding the Internet: Wireless Integrated Network Sensors," Communications of the ACM, Vol. 43, No. 5, 2000, pp. 51-58. doi: $10.1145 / 332833.332838$

[3] Y. Xu, J. Heidemann and D. Estrin, "Geography Informed Energy Conservation for Ad Hoc Routing," Proceedings of the 7th ACM/IEEE Annual International Conference on Mobile Computing and Networking, Italy, 16-21 July 2001, pp. 70-84.

[4] I. Chatzigiannakis, G. Mylonas and S. Nikoletseas, “50 Ways to Build your Application: A Survey of MiddleWare and Systems for Wireless Sensor Networks," IEEE Conference on Emerging Technologies and Factory Automation, Greece, 25-28 September 2007, pp. 466-473.

[5] K. Henricksen and R. Robinson, “A Survey of Middleware for Sensor Networks: State of the Art and Future Directions," Proceedings of the International workshop on Middleware for Sensor Networks, Melboume, 27 November-1 December 2006, pp. 60-65.

[6] D. Georgoulas and K. Blow, "In Motes Bins: A Real Time Application for Environmental Monitoring in Wireless Sensor Networks," Proceedings of the 9th IEEE/IFIP International Conference on Mobile and Wireless Communications Networks, Cork, 19-21 September 2007, pp. 21-26.doi:10.1109/ICMWCN.2007.4668173

[7] I. Chatzigiannakis, G. Mylonas and S. Nikoletseas, “jWeb
Dust: A Java-Based Generic Application Environment for Wireless Sensor Networks," Proceedings of the 1st International Conference on Distributed Computing in Sensor Systems, Marina del Rey, 30 June-1 July 2005, pp. 376-386.

[8] P. Buonadonna, D. Gay, J. Hellerstein, W. Hong and S. Madden, "TASK: Sensor Network in a Box," Proceedings of the 2nd European Workshop on Wireless Sensor Networks, Los Alamitos, January-February 2005, pp. 133-144. doi:10.1109/EWSN.2005.1462005

[9] S. Krco, D. Cleary and D. Parker, "Enabling Ubiquitous Sensor Networking over Mobile Networks through Peerto-Peer overlay Networking," Computer Communications, Vol. 28, No. 13, 2005, pp. 1586-1601.

[10] M. J. Tian, D. Zhao and W. Yan, "Backbone Based GPS-Free Localization in Mobile Ad-Hoc Networks," Chinese Journal of Electronics, Vol. 16, No.1, 2007, pp. 155-160.

[11] S. Sumathi and P. Surekha "Lab VIEW Based Advanced Instrumentation Systems,” Springer, New York, 2007.

[12] S. Ratnasamy, B. Karp, L. Yin, F. Yu, D. Estrin, R. Govindan and S. Shenker, "GHT: A Geographic Hash Table for Data-Centric Storage”, Proceedings of the 1st ACM International Workshop on Wireless Sensor Networks and Applications, Atlanta, 28 September 2002, pp.78-87.

[13] T. He, S. Krishnamurthy, J. A. Stankovic, T. Abdelzaher, L. Luo, R. Stoleru, T. Yan, L. Gu, J. Hui and B. Krogh, "Energy-Efficient Surveillance System Using Wireless Sensor Networks," Proceedings of the 2nd International Conference on Mobile Systems, Applications and Services, Boston, 6-9 June 2004, pp.270-283. doi:10.1145/990064.990096

[14] S. L. Wu, Y. C. Tseng, C. Y. Lin and J. P. Sheu, "A Multi-Channel MAC Protocol with Power Control for Multi-Hop Mobile Ad Hoc Networks,” The Computer Journal, Vol. 45, No. 1, 2002, pp. 101-110. doi:10.1093/comjnl/45.1.101

[15] R. Stoleru, H. Tian and J. A. Stankovic, "Walking GPS: A Practical Solution for Localization in Manually Deployed Wireless Sensor Networks," Proceedings of the 29th Annual IEEE International Conference on Local Computer Networks, Tampa, 16-18 November 2004, pp. 480-489.

[16] Crossbow Technology, Inc. MPR/MIB Mote Hardware Users Manual, 2006. The user manual is retrieved from http://www.xbow.com/Support/Support_pdf_files/MPRM IB_Series_Users_Manual.pdf/

[17] Crossbow Technology, Inc. Mica2 datasheet, 2006. The datasheet of Mica2 mote platform is retrieved from http://www.xbow.com/Products/Product_pdf_files/ 